Vasil Grigoriev

Independent researcher.

Belarus

\title{
Periodontal depression. The fundamental cause of periodontal disease and caries.
}

\section{Background \& Aim}

GRRGV Dental System (GD_system or GRRGV) is a system of means and methods that solve the problem of preventing periodontal disease and caries (PDC) and is used to treat PDC at any stage of development of PDC. Since 1991 the GD_system has been developing to the present. The term "periodontal depression" is introduced to indicate the state of the teeth, in which the teeth accumulate neurovascular disorders that lead to the appearance of PDC symptoms. GD_system solves the problem of preventing nerve and vascular disorders in the teeth and prevents the appearance of symptoms of PDC for a long time - dozens of years.

\section{Periodontal depression.}

The term "periodontal depression" was introduced, so it became possible to determine the state of the teeth in the period before the onset of symptoms of PDC. The methods of the GD_system make it possible to classify the state of the teeth in the period before the onset of symptoms of PDC. The system uses the means to treat the teeth in a period of periodontal depression. Elimination of periodontal depression prevents the appearance of symptoms of PDS.

Periodontal depression is the fundamental cause of periodontal disease and caries. Periodontal depression is a term that refers to the processes that occur in the tooth before the appearance of the symptoms of PDC. These are the processes of formation and accumulation of neurovascular disorders. Neurovascular disorders can occur quickly - within 3-10 days. At the initial stage, there are no signs of tooth damage. Later, these disorders accumulate, which leads to damage to the teeth by bacteria and the occurrence of other destructive processes in the tooth. There are symptoms of PDC. Bacterial lesions of the teeth are not the primary cause of the symptoms of PDC. The primary cause of PDC in the destructive processes that occur in the tooth before the onset of symptoms of PDC and which are designated as periodontal depression. GD_system allows to determine the state of the teeth in the period before the onset of symptoms of PDC and apply treatment to eliminate the pathological condition that leads to the appearance of symptoms of PDC. GD_system allows you to determine the condition of the teeth at any time and apply the treatment. When using GD_system, the symptoms of PDC do not arise because the primary cause of PDC symptoms is eliminated - periodontal 
depression. In the presence of PDC symptoms GD_system stops the destructive processes in the tooth and stabilizes the teeth for the period of application of the GD_ system. The GD_system eliminates the inevitability of the symptoms of PDC and eliminates the symptoms of PDC if they are present and does not allow them to reappear in the future. GD_system - know-how system. Used in practice from 1991 to the present. The result is the absence of symptoms of gingivitis and periodontitis at the age of 51 years. There are no new cases of caries since 1991.

\section{The fundamental cause of periodontal disease and caries.}

The fundamental cause of the symptoms of PDC is one local factor is periodontal depression. Periodontal depression forms the conditions for tooth damage from the inside. Allocations from the inside of the affected teeth are then accumulated both on the teeth and in the oral cavity. The process of tooth damage goes from the inside of the tooth and further into the oral cavity. Any other factors can cause secondary damage to the teeth, but the primary reason is one - periodontal depression. When the periodontal depression occurs, there is an accumulation of dead cells inside the tooth. Accumulation occurs due to rapid renewal of periodontal cells. Bacteria are delivered through the bloodstream. The combination of factors of the presence of bacteria in the tooth and the nutrient medium for them leads to bacterial damage to the teeth and periodontal inflammation and caries. When bacteria hit the tooth this is not the beginning of pathological processes in the teeth. The tooth is already in a pathological state - the periodontal depression. With periodontal depression, the tooth is not damaged, but it accumulates an excessive amount of dead cells, which leads to bacterial damage to the teeth. Bacterial lesions occur inside the tooth. The difficulty with the removal of dead cells from the periodontium leads to inflammation of the tooth and the appearance of symptoms of PDC. If the condition of periodontal depression is absent, the tooth is not affected by bacteria of any kind. If the periodontal depression is present, the periodontium can be affected by bacteria that do not show pathogenic activity in the normal state. The condition of periodontal depression constantly reproduces the conditions for the development of bacteria, creating a nutrient medium for their development inside the tooth. Eliminating any kind of bacteria or all bacteria on the surface of the teeth or controlling their number with antibiotics, the nutrient medium for the development of new portions of bacteria remains. Therefore, bacterial damage occurs again through the bloodstream inside the tooth or bacteria from the biofilm of the oral cavity. In the state of periodontal 
depression there is a degradation of the periodontal vascular system and a violation of the processes of renewal of periodontal cells. There is a recession of the gum. This leads to a decrease in the nutrient medium for bacteria due to slower renewal of periodontal cells and this can often be explained by the absence of caries in the presence of gum recession. Bacterial dental lesion is a secondary or concomitant process. The main pathological process of periodontium is the process of development of the periodontal depression. Bacterial damage in periodontitis is a symptom that occurs against the background of the development of periodontal depression. Traditional methods of treating periodontitis allow for a while to remove acute symptoms of periodontitis. In the future, periodontitis continues to develop as the condition of periodontal depression is not eliminated. The study of biofilm can be used to develop means for temporary elimination of periodontitis symptoms. The GD_system provides a strategic goal of ensuring dental health - ensuring nonemergence of PDC symptoms, eliminating the symptoms of PDC present and ensuring the absence of repeated relapses of periodontitis in the future. The initiator of destructive processes in the periodontium is not periodontal damage by bacteria, but nerve and vascular disorders in the periodontium. Periodontal depression is an independent disease that has not been identified so far due to a lack of funds and techniques that could be used to establish it. The GD_system provides techniques for determining the presence of a periodontal depression condition. If the destructive processes in the periodontium did not lead to significant damages of periodontal disease, the elimination of the state of periodontal depression also eliminates the bacterial damage of periodontium. The Gd_system cleans the tooth inside. Bacterial plaque is formed under the influence of secretions from within the tooth. The GD_system eliminates the conditions for tooth damage from the inside and thus eliminates the appearance of conditions for the formation of plaque with a bacterial composition that signals a bacterial lesion of periodontium.

Oral hygiene is a necessary condition for controlling bacterial infection. But the control of the bacterial composition of the biofilm is not a means of preventing the onset of symptoms of PDC. A sufficient condition for preventing the onset of symptoms of PDC is to monitor the condition of periodontal depression and eliminate it. Cleaning the teeth does not affect the primary cause of bacterial defeat of periodontium. The fundamental cause of PDC symptoms is periodontal depression. Bacterial damage is a secondary process. The progression of the neurovascular disorders in the periodontium leads to the 
appearance of periodontal pockets, to the weakening of the dentogingival attachment and the reduction of bone. Increasing periodontal pockets are colonized by bacteria and bacterial lesions of periodontal tissue occur. Even with careful oral care, teeth can be affected by gingivitis, periodontitis and caries. Elimination of bacterial dental lesions is a temporary solution to control bacterial infection, but does not solve the fundamental problem of prevention and treatment of PDC. Periodontal depression is a condition during which conditions are created for the destruction of periodontal bacteria or for the degradation of the gums. Periodontal depression is the most massive pathology to which every person is exposed, which by the age of 40-50 leads to $100 \%$ injury to people with PDC. The beginning of the development of periodontitis is due not to the action of bacteria on periodontal disease. The parodont is in a pathological state of periodontal depression, when the infection begins with bacteria. Systemic health of the body does not provide dental health. The GD_system eliminates periodontal depression and provides fundamental dental health for the period of application of the GD_system.

The picture was taken on 17.09.2018. Age 51 years. There are no symptoms of periodontitis, gingivitis. There are small damage of the enamel, which are caused by improper cleaning and the fact that important elements of the system were found in 2016. Damage to the enamel does not progress and does not cause any inconvenience. There is no hypersensitivity to sweet, sour, salty. Professional teeth cleaning and teeth whitening before taking photographs were not performed.

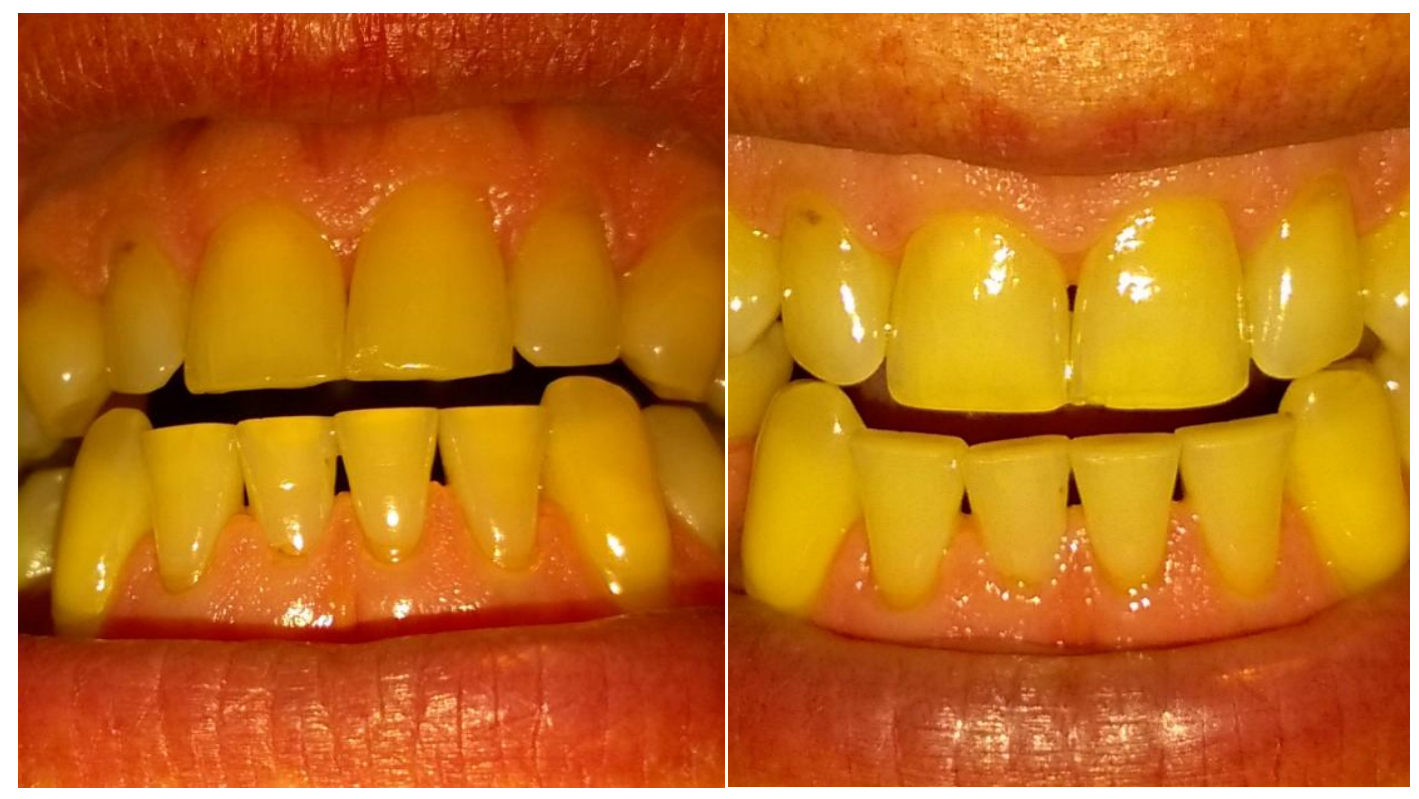


Fig.1

Fig.2

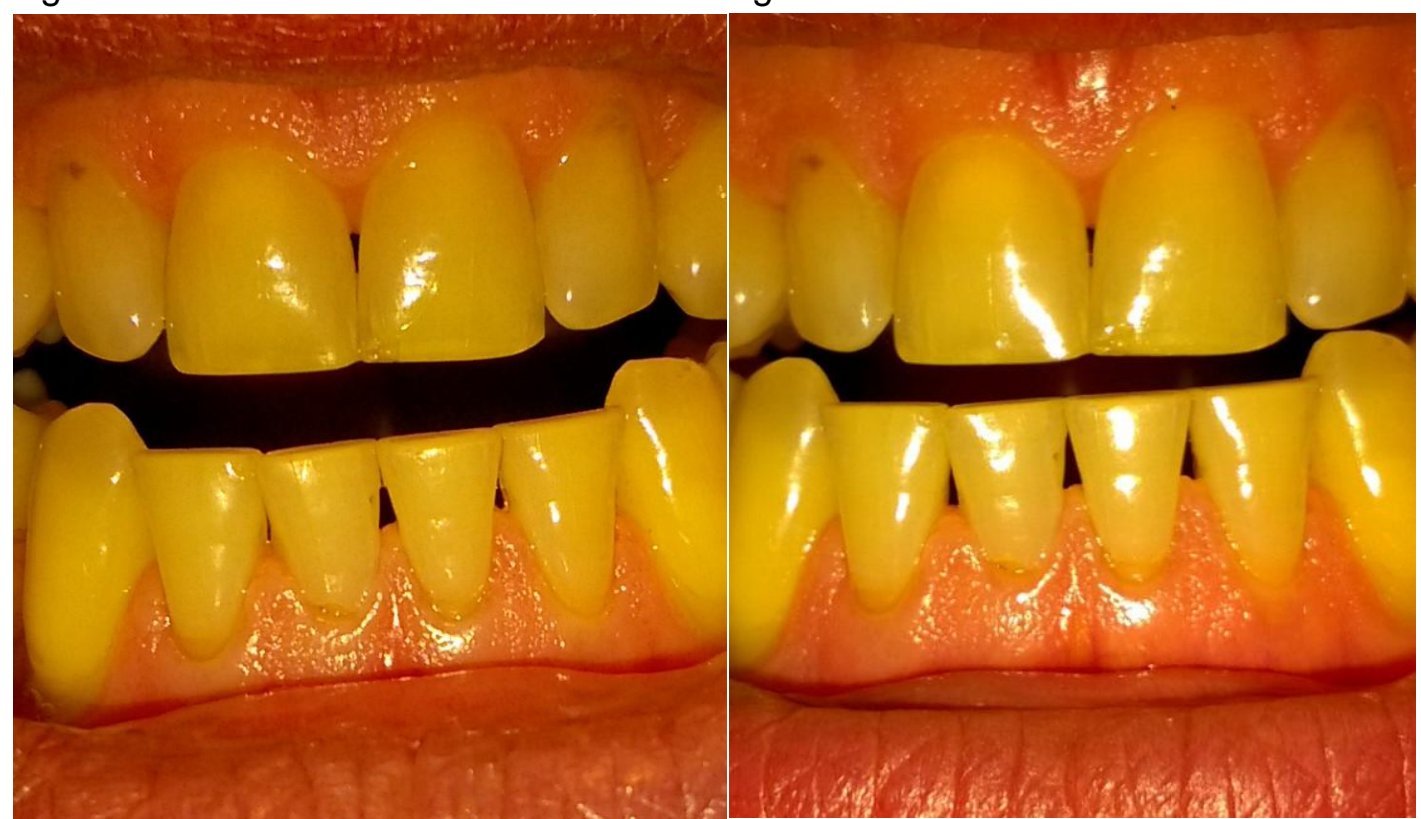

Fig.3

Fig.4
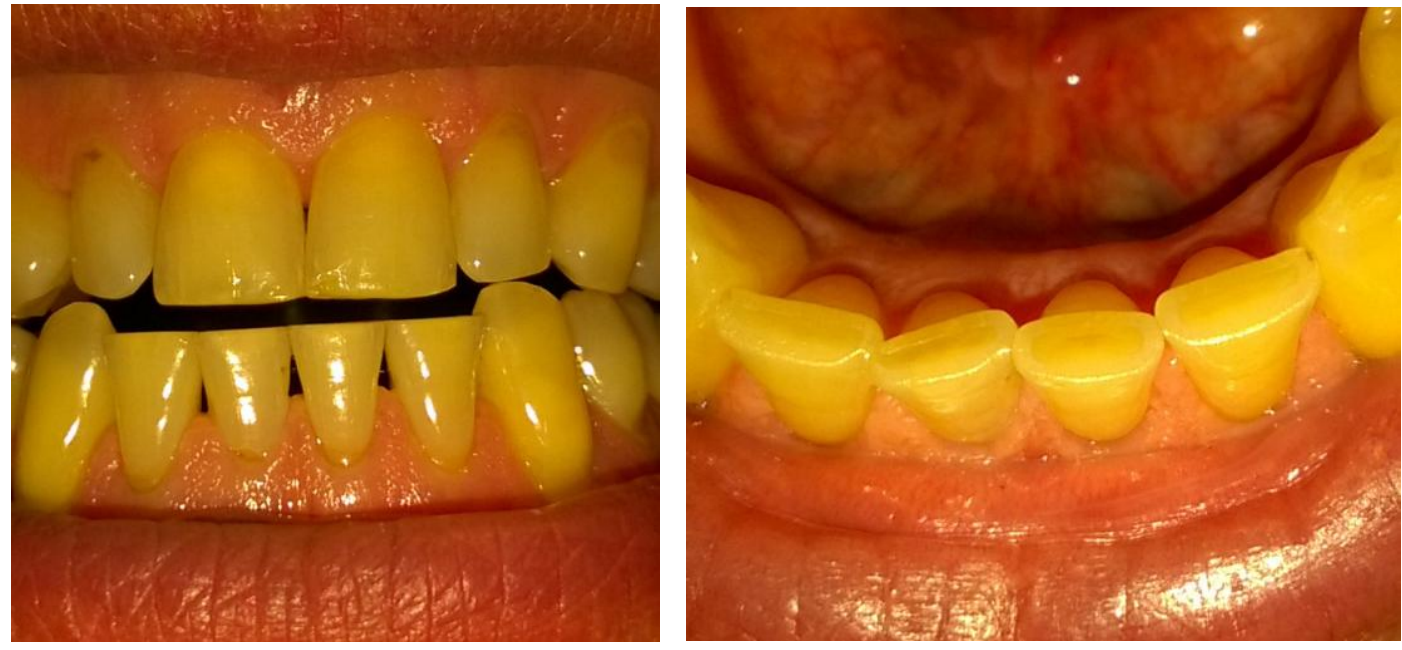

Fig.5

Fig.6

Reference

Preprint. DOI: 10.31219/osf.io/vc5a7

Causes and mechanism of development of periodontitis and caries. The relationship between periodontal diseases, depression and orofacial pain. 